gene, spastin, regulates microtubule stability to modulate synaptic structure and function. Curr Biol. 14:1135-1147.

12. Sherwood, N.T., Sun, Q., Xue, M., Zhang, B., and Zinn, K. 2004. Drosophila spastin regulates synaptic microtubule networks and is required for normal motor function. PLoS Biol. 2:e429.

13. Marques, G. 2005. Morphogens and synaptogenesis in Drosophila. J. Neurobiol. 64:417-434.

14. Jackson, A.L., et al. 2003. Expression profiling reveals off-target gene regulation by RNAi. Nat. Biotechnol. 21:635-637.

15. Sledz, C.A., Holko, M., de Veer, M.J., Silverman, R.H., and Williams, B.R. 2003. Activation of the interferon system by short-interfering RNAs. Nat. Cell Biol. 5:834-839.
16. Wittmann, C.W., et al. 2001. Tauopathy in Drosophila: neurodegeneration without neurofibrillary tangles. Science. 293:711-714.

17. Shapiro, W.R., and Young, D.F. 1984. Neurological complications of antineoplastic therapy. Acta Neurol. Scand. Suppl. 100:125-132.

18. Bonini, N.M., and Fortini, M.E. 2003. Human neurodegenerative disease modeling using Drosophila. Annu. Rev. Neurosci. 26:627-656.

19. Min, K.T., and Benzer, S. 1999. Preventing neurodegeneration in the Drosophila mutant bubblegum. Science. 284:1985-1988.

20. Ye, Y., and Fortini, M.E. 1999. Apoptotic activities of wild-type and Alzheimer's disease-related mutant presenilins in Drosophila melanogaster. J. Cell Biol. 146:1351-1364.
21. Chan, Y.B., et al. 2003. Neuromuscular defects in a Drosophila survival motor neuron gene mutant. Hum. Mol. Genet. 12:1367-1376.

22. Warrick, J.M., et al. 1998. Expanded polyglutamine protein forms nuclear inclusions and causes neural degeneration in Drosophila. Cell. 93:939-949.

23. Jackson, G.R., et al. 1998. Polyglutamine-expanded human huntingtin transgenes induce degeneration of Drosophila photoreceptor neurons. Neuron. 21:633-642.

24. Feany, M.B., and Bender, W.W. 2000. A Drosophila model of Parkinson's disease. Nature. 404:394-398.

25. Fossgreen, A., et al. 1998. Transgenic Drosophila expressing human amyloid precursor protein show gamma-secretase activity and a blistered-wing phenotype. Proc. Natl. Acad. Sci. U. S. A. 95:13703-13708.

\title{
When 7 transmembrane receptors are not G protein-coupled receptors
}

\author{
Keshava Rajagopal,1 Robert J. Lefkowitz,, ${ }^{2,3,4}$ and Howard A. Rockman',5,6 \\ ${ }^{1}$ Department of Surgery, ${ }^{2}$ Department of Medicine, ${ }^{3}$ Department of Biochemistry, ${ }^{4}$ Howard Hughes Medical Institute, \\ ${ }^{5}$ Department of Cell Biology, and ${ }^{6}$ Department of Molecular Genetics, Duke University Medical Center, Durham, North Carolina, USA.
}

\begin{abstract}
Classically, 7 transmembrane receptors transduce extracellular signals by coupling to heterotrimeric $G$ proteins, although recent in vitro studies have clearly demonstrated that they can also signal via $G$ protein-independent mechanisms. However, the physiologic consequences of this unconventional signaling, particularly in vivo, have not been explored. In this issue of the JCI, Zhai et al. demonstrate in vivo effects of G protein-independent signaling by the angiotensin II type 1 receptor (AT1R) (see the related article beginning on page 3045). In studies of the mouse heart, they compare the physiologic and biochemical consequences of transgenic cardiac-specific overexpression of a mutant AT1R incapable of $G$ protein coupling with those of a wild-type receptor. Their results not only provide the first glimpse of the physiologic effects of this newly appreciated mode of signaling but also provide important and previously unappreciated clues as to the underlying molecular mechanisms.
\end{abstract}

All vital physiologic functions of higherorder animals are critically regulated by signal transduction through 7 transmembrane receptors (7TMRs), which in the in vivo context has traditionally been understood to be mediated via heterotrimeric $G$ proteins and downstream secondmessenger molecules (1). However, for a number of years, biochemical and cellular studies have suggested that some aspects

Nonstandard abbreviations used: AT1R, angiotensin II type 1 receptor; AT1-i2m, AT1R second intracellular loop mutant; AT1-WT, WT AT1R; GRK, G protein-coupled receptor kinase; $\mathrm{Tg}$-i2 $\mathrm{m}$ mice, transgenic mice with cardiac-specific overexpression of AT1-i2m; Tg-WT mice, transgenic mice with cardiac-specific overexpression of AT1-WT; 7TMR, 7 transmembrane receptor.

Conflict of interest: The authors have declared that no conflict of interest exists.

Citation for this article: J. Clin. Invest. 115:2971-2974 (2005). doi:10.1172/JCI26950. of 7TMR-mediated signaling apparently occur independently of $\mathrm{G}$ protein activation (2). Yet, the physiologic and/or pathophysiologic roles that such novel mechanisms of signal transduction play in vivo are unknown.

Zhai et al. now provide compelling evidence for distinct physiologic consequences of $\mathrm{G}$ protein-independent signal transduction via the angiotensin II type 1 receptor (AT1R) in the heart based on their generation and study of transgenic mice with cardiac-specific overexpression of a WT AT1R (AT1-WT; Tg-WT mice) or an AT1R second intracellular loop mutant (AT1-i2m; Tg-i2m mice) (3). AT1-i2m has been shown in previous in vitro studies to be completely incapable of activating $\mathrm{G}_{\alpha} \mathrm{q}$ and $\mathrm{G}_{\alpha} \mathrm{i}$, while retaining the ability to activate molecular effectors such as Src and ERK (4). The effects of AT1-i2m overex- pression on cardiac morphology, physiology, and signal transduction were assessed and compared with those of AT1-WT.

\section{G protein-independent signal transduction via the AT1R results in ventricular hypertrophy with diminished apoptosis and a unique signaling profile} The $\mathrm{Tg}-\mathrm{i} 2 \mathrm{~m}$ mice display a pronounced cardiac phenotype, which is distinct from that of the Tg-WT mice (3). Marked ventricular dilatation and eccentric hypertrophy are present in the $\mathrm{Tg}-\mathrm{i} 2 \mathrm{~m}$ hearts to a greater extent than are observed in Tg-WT hearts. However, less cardiomyocyte apoptosis is observed in the $\mathrm{Tg}-\mathrm{i} 2 \mathrm{~m}$ hearts than in the Tg-WT hearts. These data suggest that $G$ protein-independent signal transduction via AT1-i2m results in induction of cytoprotective pathways in the heart, but that in spite of this, adverse ventricular remodeling occurs.

The $\mathrm{Tg}-\mathrm{i} 2 \mathrm{~m}$ mice also display unique electrophysiologic disturbances, as they have third-degree atrioventricular (AV) block due to impaired development of the AV node (3). Isolated cardiomyocytes from $\mathrm{Tg}-\mathrm{i} 2 \mathrm{~m}$ mice display diminished L-type $\mathrm{Ca}^{2+}$ channel currents whereas these are unimpaired in Tg-WT cells. These data point to a possible role for AT1R-mediated, $G$ protein-independent signaling in the regulation of cardiac ion channels. The authors also performed invasive hemodynamic studies that suggest a possible great- 


\section{Table 1}

Comparison between the in vivo signaling properties of the AT1-i2m and known data regarding GRK/ $\beta$-arrestin-mediated signaling

Biochemical parameter
G $_{\alpha} q$ activation
Src activation
PKC activation/translocation
Cytoplasmic phospho-ERK
Nuclear phospho-ERK
JNK activation
p38 activation
Apoptosis

AT1-i2m
Absent
Yes
Absent
Elevated
Unaffected
Absent
Absent
Decreased relative to AT1-WT

\section{GRK/3-arrestin- \\ mediated signals \\ Absent \\ Yes \\ Absent \\ Elevated \\ Unaffected \\ A \\ Absent ${ }^{B}$ \\ Decreased; PI3K, Akt, prosurvival ERK activated}

The signaling profile of AT1-i2m (middle column) and known effects of GRK/ $\beta$-arrestin-mediated signal transduction (right column) are compared for each biochemical parameter listed. AGRK/ $\beta$-arrestin-mediated signaling results in activation of JNK3 specifically (21); evidence also demonstrates that $\beta$-arrestin recruits a phosphatase that acts on JNK3, resulting in its inactivation (22). ${ }^{B}$ Activation of $\mathrm{p} 38$ by the AT1R has been shown to be $\beta$-arrestin2 independent (23), but $\beta$-arrestin2-dependent p38 activation has been reported for the chemokine receptor CXCR4 (24).

er impairment in LV systolic and diastolic function under baseline conditions in the $\mathrm{Tg}-\mathrm{i} 2 \mathrm{~m}$ mice than in Tg-WT mice; however, their echocardiographic and isolated cardiomyocyte function studies fail to confirm these findings.

The physiologic phenotype of the Tg-i2m mice is complemented by a number of noteworthy biochemical alterations, summarized in Table 1 . The biological significance of these pathways is underscored by Zhai et al.'s findings that pharmacologic inhibition of ERK and Src activity impairs AT1-i2m-mediated hypertrophy in in vitro assays (3).

\section{The AT1R signals independently of $\mathrm{G}$ proteins, but how?}

The AT1R mediates a number of essential physiologic functions in the cardiovascular system and is one of the best biochemically characterized 7TMRs with respect to G protein-independent signal transduction in vitro. Consequently, the choice of the AT1R for the physiologic study of G protein-independent signal transduction in the heart is particularly apt. However, Zhai, Sadoshima, and colleagues do not find in their current elegant studies (3) the answer to one of the most critical and important questions implicit in their work: What mechanism(s) mediate $G$ protein-independent activation of the cytoplasmic effectors Src, Ras, and ERK by AT1-i2m in vivo?

Nonetheless, their findings do offer some clues. A number of potential mechanisms for $G$ protein-independent signaling by the AT1R have been identified. Historically, the first of these was the JAK/STAT pathway. JAK2 is a tyrosine kinase that is recruited to the tail of the AT1R in an agonist-dependent fashion, leading to STAT $1 / 2$ activation (5). Additionally, the AT1R can mediate $G$ protein-independent activation of $\mathrm{Src}$, as previously shown for AT1-i2m that was used in this study (4). This results in activation of Ras and ultimately of ERK. Moreover, the activated ERK species is restricted to the cytoplasm, consistent with the current findings in vivo (3).

However, it is unclear as to what, if any, signaling molecules serve proximal functions analogous to $G$ proteins, coupling AT $1-12 m$ to activation of downstream effectors in the current study (3). Indeed, the best-characterized and best-understood proximal regulators of 7TMR signal transduction, the $G$ protein-coupled receptor kinases (GRKs) and $\beta$-arrestins, are intriguing candidates. GRKs catalyze serine/threonine phosphorylation at multiple sites in the cytoplasmic tails of 7TMRs, which promotes agonist-induced recruitment of $\beta$-arrestins to these 7TMR cytoplasmic tails (6). These actions result in the waning of $G$ protein-dependent signals, and extensive physiologic data corroborate the biochemical functions of GRKs and $\beta$-arrestins in this process, known as 7TMR desensitization (Figure 1) (6). However, recent evidence suggests that GRKs and $\beta$-arrestins also perform independent signal transducing functions. GRK/ $\beta$-arrestin-dependent signaling has been shown for a growing list of 7TMRs (reviewed in ref. 7 and references therein) as well as for other types of receptors. Thus, the GRK/ $\beta$-arrestin system is actually bifunctional, as it initiates new $G$ protein-independent signaling pathways even as it inactivates $G$ protein-mediated signaling. These $\mathrm{G}$ protein-independent signaling functions may in fact be evolutionarily conserved, as evidenced by recent data demonstrating that $\beta$-arrestin-like proteins transduce signals from a primitive 7TM $\mathrm{pH}$ sensor in fungi (8).

$\beta$-arrestin-mediated angiotensin IIstimulated activation of ERK1/2 has been extensively studied in an in vitro human embryonic kidney 293 cell system (reviewed in ref. 7). The signal appears to be carried by $\beta$-arrestin 2 , with $\beta$-arrestin 1 actually being inhibitory. As with other $\beta$-arrestinmediated signal transduction pathways studied to date, $\beta$-arrestins appear to somehow serve as scaffolds/adaptors that align the individual components of the MAPK module (Raf/MEK/ERK) as well as other upstream elements (e.g., Src, Ras) in appropriate orientation, all under the control of the agonist-occupied receptor. Recent findings further suggest that the GRK isoforms 5 and 6 play unique roles in this process whereas GRK2 may be inhibitory (9).

The in vivo biochemical findings of Zhai et al. (3) are strikingly similar to those observed in GRK/ $\beta$-arrestin-dependent signal transduction, previously documented in vitro for effectors downstream of the AT1R (Table 1). Most strikingly, cardiomyocytes from $\mathrm{Tg}$-i2m mice display cytoplasmic sequestration of phospho-ERK. This pattern of ERK phosphorylation stimulated by the AT1R recapitulates that activated by $\beta$-arrestin 2 . Interestingly, in their previous biochemical characterization of AT1-i2m in cellular transfection systems, Seta et al. demonstrated Src activation and cytosolic phospho-ERK sequestration that required the presence of an intact receptor tail (4). These data are again strongly parallel to those for $\beta$-arrestin-mediated ERK activation by several 7TMRs, which involves formation of a signaling complex including $\beta$-arrestin, Src, and ERK (10, 11). Taken together, these results suggest that the GRK/ $\beta$-arrestin system may mediate the $G$ protein-independent signaling observed in vivo by Zhai et al.

Additional similarities between the biochemical findings of Zhai et al. (3) and known hallmarks of GRK/ $\beta$-arrestindependent signal transduction are also displayed in Table 1. These include activation of cytoprotective (antiapoptotic) 


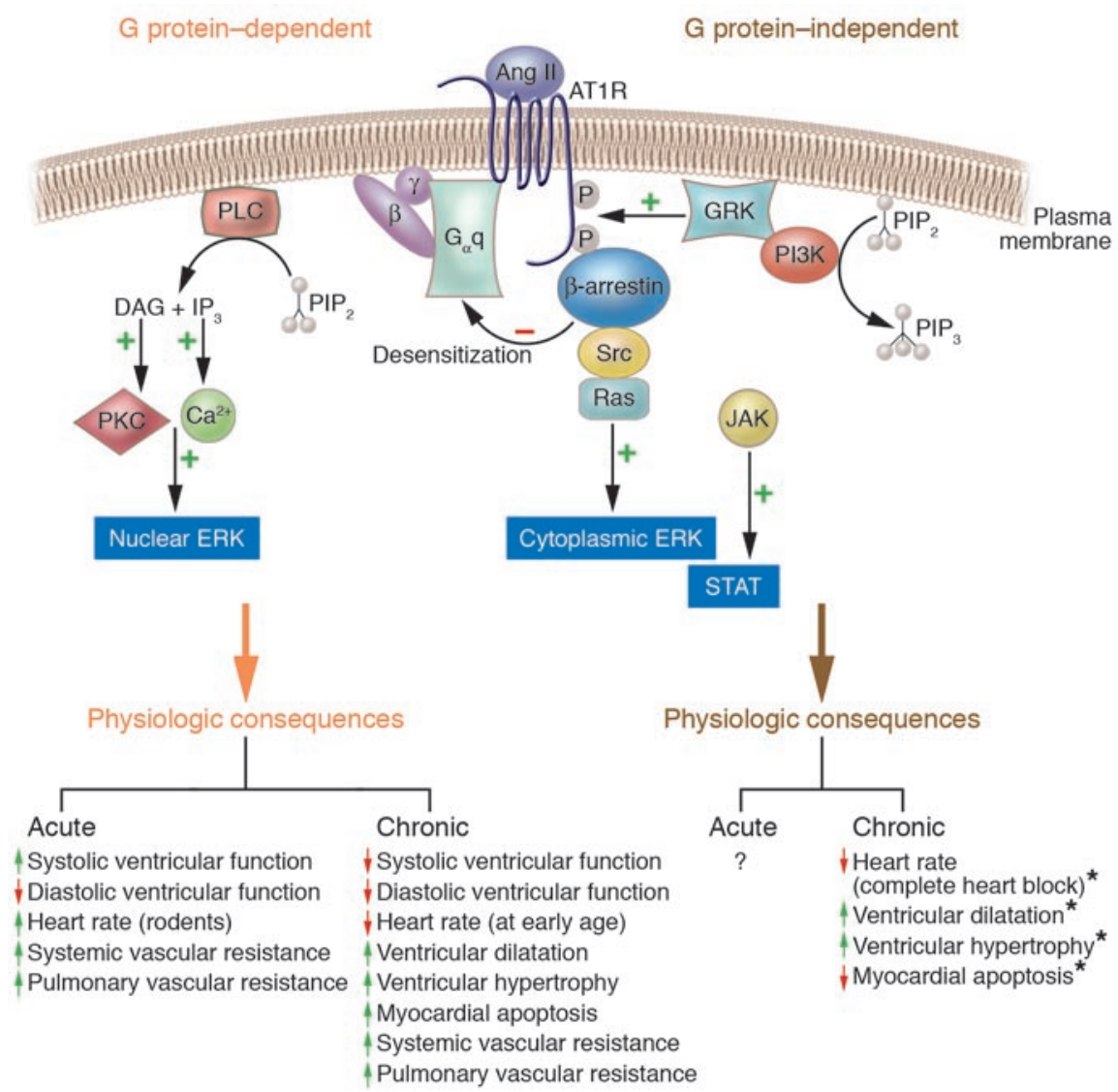

Figure 1

An integrated model of signal transduction via the AT1R and resultant physiologic effects within the cardiovascular system. Upon agonist ligand (Ang II) binding to the AT1R, both G proteindependent and $G$ protein-independent signaling pathways are activated. The AT1R is principally coupled to $G_{\alpha} q$, as illustrated, but may also couple to $G_{\alpha} i$ (not shown). Agonist-induced $\mathrm{G}_{\alpha} \mathrm{q}$ activation results in activation of phospholipase $\mathrm{C}$ (PLC), which catalyzes breakdown of the membrane lipid phosphatidylinositol-4,5-bisphosphate $\left(\mathrm{PIP}_{2}\right)$ into inositol triphosphate $\left(\mathrm{IP}_{3}\right)$ and diacylglycerol (DAG), which respectively act to increase cytosolic $\mathrm{Ca}^{2+}$ concentrations and to activate PKC. Proximally, a series of events results in the inhibition of initiation of $\mathrm{G}_{\alpha} \mathrm{q}$-mediated signaling (desensitization). Agonist-ligand binding induces GRKs to catalyze cytoplasmic serine/threonine phosphorylation, which enhances binding of $\beta$-arrestin to the AT1R cytoplasmic tail; this sterically inhibits $G_{\alpha}$ q coupling to the AT1R. $\beta$-arrestin also serves as a scaffold for signaling effectors such as Src, resulting in downstream activation of cytoplasmic ERK. Also, as illustrated, AT1R induces activation of the JAK/STAT pathway. Upon ligand stimulation, the multifunctional enzyme PI3K is recruited to the plasma membrane by interactions with GRK2 (20). In addition to its protein kinase activity, PI3K functions to catalyze the conversion of $\mathrm{PIP}_{2}$ to phoshatidylinositol-3,4,5-triphosphate ( $\left.\mathrm{PIP}_{3}\right)$ to promote 7TMR internalization (20). Both $\mathrm{G}$ protein-dependent and-independent pathways have distinct physiologic and pathophysiologic effects, as shown. The acute physiologic effects of $G$ protein-independent signaling via AT1R as well as the chronic physiologic effects of $G$ protein-independent signaling on ventricular function and systemic/pulmonary hemodynamics are unknown. The novel physiologic findings of Zhai et al. (3) are each listed and indicated with an asterisk.

pathways and failure to induce PKC activation. Recent studies have documented $\beta$-arrestin-mediated activation of several antiapoptotic signaling molecules, such as PI3K and Akt as well as prosurvival ERK, by several receptors (12-14). A model for possible physiologic outcomes of $\mathrm{G}$ protein-dependent and -independent signal transduction by the AT1R in the cardiovascular system, both acutely and chronically, is presented in Figure 1.

\section{Therapeutic implications}

The discovery of $G$ protein-independent signaling mechanisms utilized by 7TMRs presents an interesting and potentially impor- tant therapeutic opportunity. 7TMRs are, as a class, one of the most important targets of currently used drugs, both agonists and antagonists (15). However, these agents have been developed using assays that only register their ability to stimulate or inhibit G protein-dependent signals. Now, it appears that, as assessed in vitro, distinct conformations of activated AT1R and other 7TMRs are responsible for $\mathrm{G}$ protein-dependent and GRK/ $\beta$-arrestin-dependent signaling (7). These data are supported by the findings of Zhai et al. (3), who utilize a "biased receptor" that preferentially signals via G protein-independent mechanisms, presumably due to the presence of specific structural features predisposing it to $G$ protein-independent signal transduction. However, ligands may also differentially or even exclusively activate one or another signaling mechanism via a WT receptor. This phenomenon (16), termed biased agonism or ligand-directed signaling, suggests that it should be possible to develop drugs that can activate one or the other pathway preferentially.

How might this apply, for example, to the AT1R system? Although the acute physiologic effects of AT1R-mediated signal transduction on cardiovascular system function have been generally thought to be $G$ protein-mediated, the differential contributions of $G$ protein-dependent versus -independent mechanisms to these acute physiologic responses are in fact unknown. Biased AT1R agonists may help address this question, as may further studies of acute physiologic effects of signaling through biased AT1Rs such as AT1-i2m. However, in the chronic setting, several converging lines of data suggest that chronic $\mathrm{G}_{\alpha} \mathrm{q}$-driven signals are deleterious (Figure 1): (a) transgenic overexpression of $G_{\alpha} q$ in the heart results in LV dysfunction (17), and (b) generalized suppression of $\mathrm{G}_{\alpha} \mathrm{q}$-mediated signal transduction in the heart by transgenic expression of a small inhibitory peptide has been shown to prevent LV dysfunction (18) and reduce hypertrophy (19) under conditions of pressure overload. It should be possible to develop agents that antagonize this signaling (i.e., angiotensin receptor blockers [ARBs]) but which also have agonistic properties with respect to potentially beneficial or cytoprotective $G$ protein-independent signaling pathways. It is currently not known whether any of the ARBs in clinical use possess such biased agonist activity. Conversely, there may be circumstances, particularly in the acute setting, in which biased agonists incapable of inducing desensitization of 
G protein-mediated signals may be useful. Agents with pathway-selective activities might represent a potentially new generation of drugs. More studies that elucidate the physiologic roles of novel 7TMR signaling mechanisms, such as those reported here by Zhai et al. (3), will be necessary to lay the foundation for such developments.

Address correspondence to: Robert J. Lefkowitz, Howard Hughes Medical Institute, Duke University Medical Center, DUMC 3821, 467 CARL Building, Research Drive, Durham, North Carolina 27710, USA. Phone: (919) 684-2974; Fax: (919) 684-8875; E-mail: lefko001@receptor-biol.duke.edu.

1. Rockman, H., Koch, W., and Lefkowitz, R. 2002 Seven-transmembrane-spanning receptors and heart function. Nature. 415:206-212.

2. Hall, R., Premont, R., and Lefkowitz, R. 1999. Heptahelical receptor signaling: beyond the $\mathrm{G}$ protein paradigm. J. Cell Biol. 145:927-932.

3. Zhai, P., et al. 2005. Cardiac-specific overexpression of AT1 receptor mutant lacking $G_{\alpha} \mathrm{q} / \mathrm{G}_{\alpha} \mathrm{i}$ coupling causes hypertrophy and bradycardia in transgenic mice. J. Clin. Invest. 115:3045-3056. doi:10.1172/ JCI25330.

4. Seta, K., Nanamori, M., Modrall, J., Neubig, R., and Sadoshima, J. 2002. AT1 receptor mutant lacking heterotrimeric $G$ protein coupling activates the Src-Ras-ERK pathway without nuclear translocation of ERKs. J. Biol. Chem. 277:9268-9277.
5. Marrero, M., et al. 1995. Direct stimulation of Jak/ STAT pathway by the angiotensin II AT1 receptor. Nature. 375:247-250.

6. Kohout, T., and Lefkowitz, R. 2003. Regulation of $G$ protein-coupled receptor kinases and arrestins during receptor desensitization. Mol. Pharmacol. 63:9-18.

7. Lefkowitz, R., and Shenoy, S. 2005. Transduction of receptor signals by beta-arrestins. Science. 308:512-517.

8. Herranz, S., et al. 2005. Arrestin-related proteins mediate $\mathrm{pH}$ signaling in fungi. Proc. Natl. Acad. Sci. U. S. A. 102:12141-12146.

9. Kim, J., et al. 2005. Functional antagonism of different $G$ protein-coupled receptor kinases for betaarrestin-mediated angiotensin II receptor signaling. Proc. Natl. Acad. Sci. U. S. A. 102:1442-1447.

10. Luttrell, L., et al. 1999. Beta-arrestin-dependent formation of beta2 adrenergic receptor-Src protein kinase complexes. Science. 283:655-661.

11. DeFea, K., et al. 2000. The proliferative and antiapoptotic effects of substance $P$ are facilitated by formation of a beta-arrestin-dependent scaffolding complex. Proc. Natl. Acad. Sci. U. S. A. 97:11086-11091.

12. Goel, R., Phillips-Mason, P., Raben, D., and Baldassare, J. 2002. alpha-Thrombin induces rapid and sustained Akt phosphorylation by beta-arrestin1dependent and -independent mechanisms, and only the sustained Akt phosphorylation is essential for $\mathrm{G} 1$ phase progression. J. Biol. Chem. 277:18640-18648.

13. Povsic, T., Kohout, T., and Lefkowitz, R. 2003. Beta-arrestin 1 mediates insulin-like growth factor 1 (IGF-1) activation of phosphatidylinositol 3-kinase (PI3K) and anti-apoptosis. J. Biol. Chem. 278:51334-51339.

14. Revankar, C., Vines, C., Cimino, D., and Prossnitz, E. 2004. Arrestins block G protein-coupled receptor- mediated apoptosis. J. Biol. Chem. 279:24578-24584.

15. Gudermann, T., Nurnberg, B., and Schultz, G. 1995. Receptors and $G$ proteins as primary components of transmembrane signal transduction. Part 1. G protein-coupled receptors: structure and function. J. Mol. Med. 73:51-63.

16. Kenakin, T. 2003. Ligand-selective receptor conformations revisited: the promise and the problem. Trends Pharmacol. Sci. 24:346-354.

17. D’Angelo, D., et al. 1997. Transgenic Galphaq overexpression induces cardiac contractile failure in mice. Proc. Natl. Acad. Sci. U. S. A. 94:8121-8126.

18. Esposito, G., et al. 2002. Genetic alterations that inhibit in vivo pressure-overload hypertrophy prevent cardiac dysfunction despite increased wall stress. Circulation. 105:85-92.

19. Akhter, S., et al. 1998. Targeting the receptor-Gq interface to inhibit in vivo pressure overload myocardial hypertrophy. Science. 280:574-577.

20. McDonald, P., et al. 2000. Beta-arrestin2: a receptor-regulated MAPK scaffold for the activation of JNK3. Science. 290:1574-1577.

21. Willoughby, E., and Collins, M. 2005. Dynamic interaction between the dual specificity phosphatase MKP7 and the JNK3 scaffold protein beta-arrestin2. J. Biol. Chem. 280:25651-25658.

22. Hunton, D., et al. 2005. Beta-arrestin2-dependent angiotensin II type $1 \mathrm{~A}$ receptor-mediated pathway of chemotaxis. Mol. Pharmacol. 67:1229-1236.

23. Sun, Y., Cheng, Z., Ma, L., and Pei, G. 2002. Betaarrestin2 is critically involved in CXCR4-mediated chemotaxis, and this is mediated by its enhancement of p38 MAPK activation. J. Biol. Chem. 277:49212-49219.

24. Naga Prasad, S.V., Jayatilleke, A., Madamanchi, A., and Rockman, H.A. 2005. Protein kinase activity of phosphoinositide 3-kinase regulates beta-adrenergic receptor endocytosis. Nat. Cell Biol. 7:785-796.

\title{
Variable phenotypic expression of mutations in genes of the immune system
}

\author{
Rebecca H. Buckley
}

Department of Pediatrics, Duke University Medical Center, Durham, North Carolina, USA.

\begin{abstract}
Discovery of mutated genes that cause various types of primary immunodeficiencies has significantly advanced our understanding of the pathogenesis of these diseases and of the functions of normal gene products. However, it is becoming abundantly clear that the phenotypic presentation of mutations in a given gene can be quite different, depending upon the location and type of mutation but also probably upon other genetic factors and environmental influences. In this issue of the JCI, de Villartay et al. describe a third phenotype for mutations in recombination activating gene 1 (RAG1), in addition to the already known phenotypes of SCID and Omenn syndrome (see the related article on page 3291 ).
\end{abstract}

Nonstandard abbreviations used: ADA, adenosine deaminase; BTK, Bruton tyrosine kinase; CD $3 \varepsilon, C D 3 \varepsilon$ chain; RAG1, recombination activating gene 1; SAP, signaling lymphocyte activation molecule-associated (SLAM-associated) protein; WASP, Wiskott-Aldrich syndrome protein.

Conflict of interest: The author has declared that no conflict of interest exists.

Citation for this article: J. Clin. Invest. 115:2974-2976 (2005). doi:10.1172/JCI26956.
Human primary immunodeficiency diseases have been recognized for only a little over a half century (1). The spectrum of such diseases has grown at an extremely rapid pace during the past 50 years, with currently more than 120 different syndromes having been described (2). For most of that period, the conditions were identified by their clinical and immunologic presentations. How- ever, for the past 12 years, they have been defined extensively at a molecular level. Because making a firm diagnosis by clinical and immunologic criteria has always been problematic due to variability in presentation, it was thought that molecular testing would remove this ambiguity. The report by de Villartay et al. in this issue of the JCI clearly indicates that is not always the case (3). In the pre-molecular diagnostic period, there had been examples within sibships of clinical variability in expression of primary immunodeficiency. One of these was in a family reported by de Saint-Basile et al. (4) in which one sibling had Omenn syndrome and another had SCID. Subsequently, the explanation for this was found when mutations in recombination activating genes 1 and 2 (RAG1 and RAG2, respectively) were found to cause SCID (5), and later hypomorphic 